\title{
VERTEX F-ALGEBRA STRUCTURES ON THE COMPLEX ORIENTED HOMOLOGY OF H-SPACES
}

\author{
JACOB GROSS AND MARKUS UPMEIER
}

\begin{abstract}
We give a topological construction of graded vertex $F$-algebras that generalizes Joyce's vertex algebra to complex-oriented homology. Given an H-space $X$ with a $B U(1)$-action, a choice of K-theory class, and a complex oriented homology theory $E$, we build a graded vertex $F$-algebra structure on $E_{*}(X)$ where $F$ is the formal group law associated with $E$.
\end{abstract}

\section{Contents}

1. Introduction and Results

2. Formal Groups Laws and Vertex F-algebras

3. Complex Oriented Cohomology and Chern Classes

4. Proof of Theorem 1.1

5. Proof of Theorem 1.2

References

\section{INTRODUCTION AND RESUlTS}

The algebraic topology of moduli stacks, arising for example in algebraic geometry and gauge theory, is of fundamental importance for the study of invariants. Let $\mathcal{A}$ be an additive $\mathbb{C}$-linear dg-category, whose $\tau$-stable objects we wish to classify, for $\tau$ a stability condition. The category $\mathcal{A}$ has an associated moduli stack $\mathcal{M}_{\mathcal{A}}$ by [22. In [11], Joyce constructs a graded vertex algebra on the ordinary homology $H_{*}\left(\mathcal{M}_{\mathcal{A}}\right)$. Vertex algebras are algebraic structures with origins in conformal field theory which can be regarded as singular commutative rings whose operation $Y: V \otimes V \rightarrow V((z))$, the state-to-field correspondence, takes values in Laurent polynomials. This profound algebraic structure is used to describe wall-crossing formulas relating the virtual fundamental classes $\left[\mathcal{M}_{\mathcal{A}}\right]_{\tau}^{\text {virt }},\left[\mathcal{M}_{\mathcal{A}}\right]_{\tau^{\prime}}^{\text {virt }} \in H_{*}\left(\mathcal{M}_{\mathcal{A}}\right)$ for different stability conditions. These are powerful tools for computing invariants.

Motivated by physics, many authors currently investigate refined invariants such as $K$-theoretic Donaldson-Thomas invariants $[7,8,16,21$. Here the virtual classes should viewed in K-homology $K_{*}\left(\mathcal{M}_{\mathcal{A}}\right)$. As a first step towards extending wallcrossing formulas to refined invariants, we here extend Joyce's construction to any generalized (complex oriented) homology theory $E_{*}$ with associated formal group law $F(z, w)$. Our main result constructs a vertex $F$-algebra structure on $E_{*}\left(\mathcal{M}_{\mathcal{A}}\right)$ in the sense of Li [17].

In addition, our construction of vertex $F$-algebra works in greater generality, namely for any topological $\mathrm{H}$-space (i.e. abelian group up to homotopy) with an action of $B \mathrm{U}(1)$.

Date: October 1, 2021.

2000 Mathematics Subject Classification. 17B69, 55N20.

Key words and phrases. Vertex algebra, formal group law, generalized cohomology, $H$-space. 
Let $E^{*}$ be a complex oriented generalized cohomology theory with associated formal group law $F(z, w)$ over its coefficient ring $R_{*}$, see 33 . As a preliminary result, we present a Laurent-polynomial version of the Conner-Floyd Chern classes (see Definition 3.4) with values in $E^{*}$.

Theorem 1.1. For every class $\theta \in K^{0}(X)$ in the topological $K$-theory of topological space $X$ there is an $R$-linear transformation

$$
(-) \cap C_{z}^{E}(\theta): E_{*}(X) \longrightarrow E_{*}(X) \llbracket z \rrbracket\left[z^{-1}\right] \quad a \longmapsto a \cap C_{z}^{E}(\theta),
$$

of degree $-2 r$ if $\theta$ has constant rank $r \in \mathbb{Z}$, with the following properties:

(a) (Naturality.) For continuous $f: X^{\prime} \rightarrow X, \theta \in K^{0}(X)$, and $a^{\prime} \in E_{*}\left(X^{\prime}\right)$

$$
f_{*}\left(a^{\prime} \cap C_{z}^{E}\left(f^{*}(\theta)\right)\right)=f_{*}\left(a^{\prime}\right) \cap C_{z}^{E}(\theta) .
$$

(b) (Direct sums.) For $\zeta, \theta \in K^{0}(X)$ and $a \in E_{*}(X)$ we have

$$
a \cap C_{z}^{E}(\zeta+\theta)=\left[a \cap C_{z}^{E}(\zeta)\right] \cap C_{z}^{E}(\theta) .
$$

(c) (Normalization.) For a complex line bundle $L \rightarrow X$ and $a \in E_{*}(X)$ we have

$$
a \cap C_{z}^{E}(L)=a \cap F\left(z, c_{1}^{E}(L)\right) .
$$

More generally, for any $\theta \in K^{0}(X)$ we have

$$
a \cap C_{z}^{E}(L \otimes \theta)=i_{z, c_{1}^{E}(L)}\left(a \cap C_{F\left(z, c_{1}^{E}(L)\right)}^{E}(\theta)\right) .
$$

Here, as usual, the variable $z$ has degree -2 . We prove Theorem 1.1] in 4 . The notations used in (1.4) and (1.5) will be explained in Notations 3.9 \& 4.1 below.

For our main result, let $X$ be an H-space with an operation $\Phi: X \times X \rightarrow X$ that is associative, commutative, and has a unit $e \in X$ up to homotopy. Recall that the classifying space $B \mathrm{U}(1)$ for complex line bundles is an H-space with the tensor product $\mu_{B \mathrm{U}(1)}$ and trivial bundle $e_{B \mathrm{U}(1)}$. Assume there is an action $\Psi$ of $B \mathrm{U}(1)$ on $X$ up to homotopy, meaning $\Psi \circ\left(\operatorname{id}_{B \mathrm{U}(1)} \times \Psi\right) \simeq \Psi \circ\left(\mu_{B \mathrm{U}(1)} \times \mathrm{id}_{X}\right)$ and $\Psi\left(e_{B \mathrm{U}(1)},-\right) \simeq \operatorname{id}_{X}$. Suppose $\Psi(e,-) \simeq e$ is an h-fixed point and $\Phi \circ(\Psi \times \Psi) \circ \delta \simeq$ $\Psi \circ\left(\Phi \times \mathrm{id}_{B \mathrm{U}(1)}\right)$, where $\delta\left(x_{1}, x_{2}, g\right)=\left(x_{1}, g, x_{2}, g\right)$. The set of connected components $\pi_{0}(X)$ is a monoid with unit $\Omega=[e]$ and operation $\alpha+\beta=\Phi_{*}(\alpha \otimes \beta)$ and we partition $X=\coprod_{\alpha \in \pi_{0}(X)} X_{\alpha}$. Write $\Phi_{\alpha, \beta}: X_{\alpha} \times X_{\beta} \rightarrow X_{\alpha+\beta}, \Psi_{\alpha}: B \mathrm{U}(1) \times X_{\alpha} \rightarrow$ $X_{\alpha}$ for the restrictions. Let $\theta_{\alpha, \beta} \in K^{0}\left(X_{\alpha} \times X_{\beta}\right)$ for all $\alpha, \beta$.

Theorem 1.2. Given $(X, \Phi, e, \Psi)$ as above, suppose the following identities hold for all $\alpha, \beta, \gamma \in \pi_{0}(X)$ :

$$
\begin{aligned}
\left(\Phi_{\alpha, \beta} \times \operatorname{id}_{X_{\gamma}}\right)^{*}\left(\theta_{\alpha+\beta, \gamma}\right) & =\pi_{\alpha, \gamma}^{*}\left(\theta_{\alpha, \gamma}\right)+\pi_{\beta, \gamma}^{*}\left(\theta_{\beta, \gamma}\right), \\
\left(\operatorname{id}_{X_{\alpha}} \times \Phi_{\beta, \gamma}\right)^{*}\left(\theta_{\alpha, \beta+\gamma}\right) & =\pi_{\alpha, \beta}^{*}\left(\theta_{\alpha, \beta}\right)+\pi_{\alpha, \gamma}^{*}\left(\theta_{\alpha, \gamma}\right), \\
\left(\Psi_{\alpha} \times \operatorname{id}_{X_{\beta}}\right)^{*}\left(\theta_{\alpha, \beta}\right) & =\pi_{B \mathrm{U}(1)}^{*}(\mathcal{L}) \otimes \pi_{\alpha, \beta}^{*}\left(\theta_{\alpha, \beta}\right), \\
\left(\operatorname{id}_{X_{\alpha}} \times \Psi_{\beta}\right)^{*}\left(\theta_{\alpha, \beta}\right) & =\pi_{B \mathrm{U}(1)}^{*}(\mathcal{L})^{\vee} \otimes \pi_{\alpha, \beta}^{*}\left(\theta_{\alpha, \beta}\right), \\
\left.\theta\right|_{X_{\alpha} \times\{\Omega\}} & =0,\left.\quad \theta\right|_{\{\Omega\} \times X_{\beta}}=0, \\
\sigma^{*}\left(\theta_{\beta, \alpha}\right) & =\left(\theta_{\alpha, \beta}\right)^{\vee} .
\end{aligned}
$$

Here $\sigma$ swaps the factors of $X_{\alpha} \times X_{\beta}$ and $\mathcal{L} \rightarrow B \mathrm{U}(1)$ is the universal line bundle with dual $\mathcal{L}^{\vee}$. With the $F$-shift operator $\mathcal{D}(z)$ of (3.3) below, the graded $R_{*}$-module

$$
V_{*}=\bigoplus_{\alpha \in \pi_{0}(X)} E_{*-\operatorname{rk} \theta_{\alpha, \alpha}}\left(X_{\alpha}\right)
$$

is a graded nonlocal vertex $F$-algebra $\left(V_{*}, \mathcal{D}, \Omega, Y\right)$ with state-to-field correspondence

$$
Y(a, z) b=\left(\Phi_{\alpha, \beta}\right)_{*}\left(\mathcal{D}_{\alpha}(z) \otimes \operatorname{id}_{E_{*}\left(X_{\beta}\right)}\right)\left[(a \otimes b) \cap C_{z}^{E}\left(\theta_{\alpha, \beta}\right)\right] .
$$


Similarly, the graded $R_{*}$-module

$$
\bar{V}_{*}=\bigoplus_{\alpha \in \pi_{0}(X)} E_{*-2 \operatorname{rk} \theta_{\alpha, \alpha}}\left(X_{\alpha}\right)
$$

becomes a graded vertex $F$-algebra $\left(\bar{V}_{*}, \mathcal{D}, \Omega, \bar{Y}\right)$, where

$$
\bar{Y}(a, z) b=\left(\Phi_{\alpha, \beta}\right)_{*}\left(\mathcal{D}_{\alpha}(z) \otimes \operatorname{id}_{E_{*}\left(X_{\beta}\right)}\right)\left[(a \otimes b) \cap \bar{C}_{z}^{E}\left(\theta_{\alpha, \beta}\right)\right]
$$

uses the operation of degree $-4 \operatorname{rk} \theta_{\alpha, \beta}$ defined by

$$
c \cap \bar{C}_{z}^{E}\left(\theta_{\alpha, \beta}\right)=\left[c \cap C_{z}^{E}\left(\theta_{\alpha, \beta}\right)\right] \cap C_{\iota(z)}^{E}\left(\sigma^{*}\left(\theta_{\beta, \alpha}\right)\right), \quad c \in E_{*}\left(X_{\alpha} \times X_{\beta}\right) .
$$

Here $\iota(z)$ is the inverse for $F$ (see $\$ 2$ ). The proof of Theorem 1.2 is given in $\$ 5$

As a special case, our result applies to the topological realization $X=\mathcal{M}_{\mathcal{A}}^{\text {top }}$ of a moduli stack. Taking direct sums in the additive category defines $\Phi$ making $\mathcal{M}_{\mathcal{A}}$ into an H-space. Moreover, scaling morphism by $\mathrm{U}(1)$ defines an operation $\Psi$ of the quotient stack $[* / / \mathrm{U}(1)]$, endowing $\mathcal{M}_{\mathcal{A}}^{\text {top }}$ with the required action of $B U(1)=[* / / \mathrm{U}(1)]^{\text {top }}$. As shown in Proposition 3.3 below, this action yields an $F$ shift operator $\mathcal{D}(z)$. The $K$-theory classes $\theta_{\alpha, \beta}$ are given by the Ext-complexes in the dg-category $\mathcal{A}$, which satisfy (1.6)-11.11). In geometric examples, one may wish to incorporate signs $\epsilon_{\alpha, \beta}$ into (1.15). These are related to orientations, see [11, §8.3]. The orientation problems were solved in the series 12 14. For simplicity, we ignore this additional data here and set up a symmetrized construction without signs.

\section{Formal Groups Laws and Vertex F-ALgebras}

Notation 2.1. $-R_{*}=R^{-*}$ a graded commutative ring with unit

- $V_{*}$ a graded module over $R_{*}$

- $z, w$ variables of degree -2

- $F(z, w)$ a graded formal group law over $R_{*}$

- $V \llbracket z \rrbracket$ the formal power series $\sum_{i=0}^{\infty} a_{i} z^{i}$; a ring when $V=R$

- $V((z))$ the group of Laurent series $\sum_{i=-\infty}^{+\infty} a_{i} z^{i}$ with its partially defined product. The fact that $V((z))$ is not a ring frequently causes confusion.

- The meromorphic series $V \llbracket z \rrbracket\left[z^{-1}\right]$; a ring when $V=R$.

- $i_{z, w}: V \llbracket z, w \rrbracket\left[z^{-1}, w^{-1}, F(z, w)^{-1}\right] \rightarrow V((z, w))$ expands $F(z, w)^{-N}$, see Notation 2.4. We have $i_{z, w}\left(V \llbracket z, w \rrbracket\left[F(z, w)^{-1}\right]\right) \subset V((z)) \llbracket w \rrbracket$.

- $(-1)^{a}$ means $(-1)^{\operatorname{degree}(a)}$

Definition 2.2. A graded formal group law over $R_{*}$ is a formal power series $F(z, w)=\sum_{i, j \geqslant 0} F_{i j} z^{i} w^{j} \in R \llbracket z, w \rrbracket$ with $F_{i j} \in R_{2 i+2 j-2}$ satisfying

$$
F(z, w)=F(w, z), \quad F(z, 0)=z, \quad F(F(z, w), v)=F(z, F(w, v)) .
$$

There exists a unique power series $\iota \in R \llbracket z \rrbracket$ with $F(z, \iota(z))=0$, the inverse. Note that $\iota(\iota(z))=z$ and $\iota(F(\iota(z), w))=F(z, \iota(w))$.

Example 2.3. (i) The additive formal group law $\mathbb{G}_{\mathrm{a}}$ over $\mathbb{Z}$ (in degree zero) is defined by $F(z, w)=z+w$, and the inverse is $\iota(z)=-z$.

(ii) The multiplicative formal group law $\mathbb{G}_{\mathrm{m}}$ over $\mathbb{Z}$ is defined by $F(z, w)=z+$ $w+z w$ and has $\iota(z)=(1+z)^{-1}-1=-z+z^{2}-z^{3}+\cdots$.

(iii) There is a universal formal group law $\mathbb{G}_{\mathrm{u}}$ over the Lazard ring $R_{L}$ generated by variables $F_{i j}$ subject to the relations contained in (2.1). 
Notation 2.4. It follows from (2.1) that for a general formal group law

$$
F(z, w)=z+w+O(z w), \quad \iota(z)=-z+O\left(z^{2}\right) .
$$

Write $F(z, w)=z(1+w / z+w G(z, w))$ and expand using the binomial theorem

$$
i_{z, w} F(z, w)^{n}=\sum_{k=0}^{\infty}\left(\begin{array}{l}
n \\
k
\end{array}\right) z^{n-k} w^{k}(1+z G(z, w))^{k} \in R \llbracket w \rrbracket((z)), \quad n \in \mathbb{Z} .
$$

As the $k$-th summand has $w$-degree $\geqslant k$, this converges as a formal power series. Define $i_{w, z} F(z, w)^{n} \in R \llbracket z \rrbracket((w))$ by expanding $F(z, w)=w(1+z / w+z G(z, w))$ similarly. We extend $i_{z, w}$ and $i_{w, z}$ to $V \llbracket z, w \rrbracket\left[z^{-1}, w^{-1}\right]\left[F(z, w)^{-1}\right]$ by linearity.

Note that $i_{z, w} F(z, w)^{n} \cdot F(z, w)^{n}=1$ and $i_{w, z} F(w, z)^{n} \cdot F(z, w)^{n}=1$. For every $P(z, w)=\sum_{n \geqslant-N} a_{n}(z, w) F(z, w)^{n} \in V \llbracket z, w \rrbracket\left[z^{-1}, w^{-1}\right]\left[F(z, w)^{-1}\right]$ we thus have

$$
F(z, w)^{N}\left(i_{z, w} P(z, w)-i_{w, z} P(z, w)\right)=0 .
$$

Definition 2.5. Let $V_{*}$ be a graded $R_{*}$-module and $F$ a graded formal group law over $R_{*}$. An $F$-shift operator is a graded $R$-linear map $\mathcal{D}(z): V \rightarrow V \llbracket z \rrbracket$ with

$$
\mathcal{D}(0)=\operatorname{id}_{V}, \quad \mathcal{D}(z) \circ \mathcal{D}(w)=\mathcal{D}(F(z, w)) .
$$

Example 2.6. Let $R=\mathbb{Q}, V=\mathbb{Q}[w]$. Then $\mathcal{D}(z)(f(w))=e^{z \frac{d}{d w}} f(w)$ defines a $\mathbb{G}_{\mathrm{a}}$-shift operator. The relation $\mathcal{D}(z)(f(w))=f(z+w)$ motivates the terminology.

We now define vertex $F$-algebras. For $F=\mathbb{G}_{\mathrm{a}}$ we recover ordinary vertex algebras, see Frenkel-Ben-Zvi [5], Frenkel-Lepowsky-Meurman [6], and Kac [15].

Definition 2.7. Let $F(z, w)$ be a graded formal group law over $R_{*}$. A graded nonlocal vertex $F$-algebra is a graded $R_{*}$-module $V_{*}$, a vacuum vector $\Omega \in V_{0}$, an $F$-shift operator $\mathcal{D}(z)$, and a graded $R$-linear state-to-field correspondence

$$
V \otimes_{R} V \longrightarrow V \llbracket z \rrbracket\left[z^{-1}\right], \quad a \otimes b \longmapsto Y(a, z) b,
$$

satisfying the following axioms:

(a) Vacuum and creation: $Y(a, z) \Omega$ is holomorphic for all $a \in V$ and

$$
\begin{aligned}
\left.Y(a, z) \Omega\right|_{z=0} & =a, \\
Y(\Omega, z) & =\operatorname{id}_{V} .
\end{aligned}
$$

(b) F-translation covariance: for all $a \in V$ we have

$$
\begin{aligned}
Y(\mathcal{D}(w)(a), z) & =i_{z, w} Y(a, F(z, w)), \\
\mathcal{D}(z) \Omega & =\Omega .
\end{aligned}
$$

(c) Weak F-associativity: for all $a, b, c \in V$ there exists $N \geqslant 0$ with

$$
F(z, w)^{N} Y(Y(a, z) b, w) c=F(z, w)^{N} i_{z, w} Y(a, F(z, w)) Y(b, w) c .
$$

A graded nonlocal vertex $F$-algebra is a graded vertex $F$-algebra if, in addition,

$$
Y(a, z) b=(-1)^{a b} \mathcal{D}(z) \circ Y(b, \iota(z)) a, \quad \text { for all } a, b \in V .
$$

Remark 2.8. It is a consequence of (2.6) (2.11) that for all $a, b, c \in V$ there exists $N \geqslant 0$ with

$$
(z-w)^{N} Y(a, z) Y(b, w) c=(-1)^{a b}(z-w)^{N} Y(b, w) Y(a, z) c .
$$

So our definitions agree with those given by Li [17] in the ungraded case. 


\section{Complex Oriented Cohomology and Chern Classes}

Let $E^{*}$ be a generalized cohomology theory, see for example Rudyak [19, Ch. II, $\S 3]$. Thus, for every pair $A \subset X$ of topological spaces there is defined a graded abelian group $E^{*}(X, A)$. Continuous maps $f:(X, A) \rightarrow\left(X^{\prime}, A^{\prime}\right)$ induce homomorphisms $f^{*}: E^{*}\left(X^{\prime}, A^{\prime}\right) \rightarrow E^{*}(X, A)$ that depend only on the homotopy class of $f$. For a pointed space $x_{0} \in X$ write $\widetilde{E}^{*}(X)=E^{*}\left(X,\left\{x_{0}\right\}\right)$ for reduced cohomology. The smash product of $\left(X, x_{0}\right)$ and $\left(Y, y_{0}\right)$ is the quotient $X \wedge Y=(X \times Y) /(X \vee Y)$ with one-point union $X \vee Y=\left(X \times\left\{y_{0}\right\}\right) \cup\left(\left\{x_{0}\right\} \times Y\right)$ collapsed to become the new base-point. As part of the structure, $E^{*}$ comes equipped with natural suspension isomorphisms $\sigma_{X}: \widetilde{E}^{*}(X) \rightarrow \widetilde{E}^{*+1}\left(X \wedge \mathcal{S}^{1}\right)$.

Suppose $E^{*}$ is a multiplicative generalized cohomology theory. Then there is a bilinear cross product $\otimes: E^{*}(X, A) \otimes E^{*}(Y, B) \rightarrow E^{*}(X \times Y, X \times B \cup A \times Y)$ and units $1_{X} \in E^{0}(X)$, both natural. Pulling the cross product back along the diagonal makes $E^{*}(X)$ a graded commutative unital $R^{*}$-algebra for the cup product ' $\cup$ ' over the coefficient ring $R^{*}=E^{*}(\mathrm{pt})$. Dually, there is a homological cross product that in particular makes $E_{*}(X)$ a graded module over $R_{*}=R^{-*}$. There is a cap product

$$
E_{a}(X) \otimes_{R} E^{b}(X) \longrightarrow E_{a-b}(X), \quad a \otimes \varphi \mapsto a \cap \varphi
$$

which is $R$-linear, unital $a \cap 1=a$, and natural $f_{*}\left(a \cap f^{*}\left(\varphi^{\prime}\right)\right)=f_{*}(a) \cap \varphi^{\prime}$, where $f: X \rightarrow X^{\prime}$ and $\varphi^{\prime} \in E^{b}\left(X^{\prime}\right)$. See Rudyak [19] for further properties.

Definition 3.1. The suspension isomorphism shows that $\widetilde{E}^{*}\left(\mathbb{C P}^{1}\right) \cong \widetilde{E}^{*}\left(\mathcal{S}^{2}\right) \cong$ $R^{*-2}$ is a free $R$-module on a single generator. A multiplicative cohomology theory $E^{*}$ is complex orientable if $i^{*}: \widetilde{E}^{*}\left(\mathbb{C P}^{\infty}\right) \rightarrow \widetilde{E}^{*}\left(\mathbb{C P}^{1}\right)$ is surjective, where $\mathbb{C P}^{\infty} \cong \operatorname{colim}_{m} \mathbb{C P}^{m}$ and $i: \mathbb{C P}^{1} \hookrightarrow \mathbb{C P}^{\infty}$. A complex orientation is a choice of $\xi_{E} \in \widetilde{E}^{2}\left(\mathbb{C} \mathbb{P}^{\infty}\right)$ such that $i^{*}\left(\xi_{E}\right)$ generates the $R$-module $\widetilde{E}^{*}\left(\mathbb{C P}^{1}\right)$.

The presence of the permanent cycle $\left.\xi_{E}\right|_{\mathbb{C P} m}$ implies that the Atiyah-Hirzebruch spectral sequence $H^{p}\left(\mathbb{C P}^{m} ; E^{q}(\mathrm{pt})\right) \Longrightarrow E^{p+q}\left(\mathbb{C P}^{m}\right)$ collapses, see Adams [1, p. 42]. Hence we have canonical isomorphisms

$$
E^{*}\left(\mathbb{C P}^{m}\right) \cong R\left[\xi_{E}\right] /\left(\xi_{E}^{m+1}\right), \quad E^{*}\left(\mathbb{C P}^{\infty}\right) \cong \lim E^{*}\left(\mathbb{C P}^{m}\right) \cong R \llbracket \xi_{E} \rrbracket .
$$

More generally, let $P \rightarrow X$ be a bundle of projective spaces $\mathbb{C P}^{m}$ and suppose that $w \in E^{*}(P)$ restricts on every fiber $P_{x}$ to generators $1_{P_{x}},\left.w\right|_{P_{x}}, \ldots,\left.w^{m}\right|_{P_{x}}$ of the $R$ module $E^{*}\left(P_{x}\right)$. Then Dold's theorem implies that $E^{*}(P)$ is a free $E^{*}(X)$-module on $1_{P}, w, \ldots, w^{m}$, see [4, (7.4)]. In particular,

$$
E^{*}\left(X \times \mathbb{C P}^{\infty}\right) \cong E^{*}(X) \llbracket \xi_{E} \rrbracket, \quad E^{*}\left(\mathbb{C P}^{\infty} \times \mathbb{C P}^{\infty}\right) \cong R \llbracket \pi_{1}^{*}\left(\xi_{E}\right), \pi_{2}^{*}\left(\xi_{E}\right) \rrbracket .
$$

Definition 3.2. Let $\xi_{E}$ be a complex orientation of $E^{*}$. Write $\mathcal{L} \rightarrow \mathbb{C P}^{\infty}$ for the universal complex line bundle with $\left.\mathcal{L}\right|_{L}=L$. Recall that $\mathbb{C P}^{\infty}=B \mathrm{U}(1)$ is an $\mathrm{H}$ space with operation a classifying map $\mu_{\mathbb{C P} \infty}: \mathbb{C P}^{\infty} \times \mathbb{C P}^{\infty} \rightarrow \mathbb{C P}^{\infty}$ of the tensor product $\pi_{1}^{*}(\mathcal{L}) \otimes \pi_{2}^{*}(\mathcal{L})$ and unit $t_{0}$ the trivial line bundle. The associated formal group law $F=\sum_{i, j \geqslant 0} F_{i j} z^{i} w^{j}$ is defined by the expansion

$$
\mu_{\mathbb{C P} \infty}^{*}\left(\xi_{E}\right)=\sum_{i, j \geqslant 0} F_{i j} \xi_{E}^{i} \otimes \xi_{E}^{j}, \quad F_{i j} \in R^{2-2 i-2 j}=R_{2 i+2 j-2} .
$$

As in [1, p. 42] the homology $E_{*}\left(\mathbb{C P}^{\infty}\right)$ is the free $R$-module on the dual generators $t_{n}, n \geqslant 0$, of degree $2 n$ characterized by $\left\langle t_{n}, \xi_{E}^{m}\right\rangle=\delta_{n}^{m}$.

Proposition 3.3. Let $\left(E^{*}, \xi_{E}\right)$ be a complex oriented cohomology theory and associated formal group law $F(z, w)$. Suppose $\Psi: B U(1) \times X \rightarrow X$ satisfies the axioms for a group action of the $H$-space $B \mathrm{U}(1)$ on $X$ up to homotopy. Then

$$
\mathcal{D}(z)(a)=\sum_{k \geqslant 0} \Psi_{*}\left(t_{k} \otimes a\right) z^{k}, \quad a \in E_{*}(X),
$$

defines an $F$-shift operator on $E_{*}(X)$. 
Proof. Since $\Psi\left(t_{0}, x\right)=x$ is neutral, $\mathcal{D}(0)=\operatorname{id}_{E_{*}(X)}$. Define coefficients $F_{i j}^{n}$ by $F(z, w)^{n}=\sum_{i, j \geqslant 0} F_{i j}^{n} z^{i} w^{j}$. Then $\left(\mu_{\mathbb{C P} \infty}\right)_{*}\left(t_{i} \otimes t_{j}\right)=\sum_{n \geqslant 0} F_{i j}^{n} t_{n}$, and so

$$
\begin{aligned}
\mathcal{D}(z) \circ \mathcal{D}(w)(a) & =\sum_{i, j \geqslant 0} \Psi_{*}\left(t_{i} \otimes \Psi_{*}\left(t_{j} \otimes a\right)\right) z^{i} w^{j} \\
& =\sum_{i, j \geqslant 0} \Psi_{*}\left(\left(\mu_{\mathbb{C P} \infty}\right)_{*}\left(t_{i} \otimes t_{j}\right) \otimes a\right) z^{i} w^{j} \\
& =\sum_{i, j, n \geqslant 0} \Psi_{*}\left(t_{n} \otimes a\right) F_{i j}^{n} z^{i} w^{j}=\mathcal{D}(F(z, w)) .
\end{aligned}
$$

Definition 3.4. Let $V \rightarrow X$ be a complex vector bundle of rank $n$ with zero section $0_{X}$. The bundle of projective spaces $\mathbb{P}(V)=\left(V \backslash 0_{X}\right) / \mathbb{C}^{*}$ carries a tautological line bundle $\mathcal{L}_{V} \rightarrow \mathbb{P}(V)$ with $\left.\mathcal{L}_{V}\right|_{L}=L$. Its classifying map $f_{\mathcal{L}_{V}}: \mathbb{P}(V) \rightarrow \mathbb{C P}^{\infty}$ is unique up to homotopy. Define $w=f_{\mathcal{L}_{V}}^{*}\left(\xi_{E}\right)$ using the complex orientation. By the above, $E^{*}(\mathbb{P}(V))$ is a free $E^{*}(X)$-module with basis $1_{\mathbb{P}(V)}, w, \ldots, w^{n-1}$. The Conner-Floyd Chern classes are defined by expanding $w^{n}$ in this basis:

$$
c_{0}^{E}(V)=1, \quad 0=\sum_{i=0}^{n}(-1)^{i} c_{i}^{E}(V) \cdot w^{n-i}, \quad c_{i}^{E}(V)=0(\forall i>n)
$$

Naturality under pullback is obvious. There is a Whitney sum formula [4, p. 47]

$$
c_{k}^{E}(V \oplus W)=\sum_{i=0}^{k} c_{i}^{E}(V) c_{k-i}^{E}(W) .
$$

For complex line bundles $\mathcal{L}_{L} \rightarrow \mathbb{P}(L)$ is isomorphic to $L \rightarrow X$ so $c_{1}^{E}(L)=f_{L}^{*}\left(\xi_{E}\right)$ for the classifying map $f_{L}$ of $L$. In particular,

$$
c_{1}^{E}\left(L_{1} \otimes L_{2}\right)=F\left(c_{1}^{E}\left(L_{1}\right), c_{1}^{E}\left(L_{2}\right)\right) .
$$

Moreover, $c_{1}^{E}(\underline{\mathbb{C}})=0$ as $\xi_{E}$ is reduced. Hence $c_{i}^{E}\left(\underline{\mathbb{C}}^{N}\right)=0$ for every trivial bundle.

Example 3.5. Ordinary cohomology $E^{*}=H^{*}$ has a complex orientation $\xi_{H}$ in $H^{2}\left(\mathbb{C P}^{\infty}\right)=\lim H^{2}\left(\mathbb{C P}^{m}\right)$ that is Poincare dual to the fundamental class $\left[\mathbb{C P}^{m-1}\right] \in$ $H_{m-2}\left(\mathbb{C P}^{m}\right)$ with orientation of $\mathbb{C P}^{m-1}$ fixed by the complex structure. We obtain the ordinary Chern classes, and $c_{1}\left(L_{1} \otimes L_{2}\right)=c_{1}\left(L_{1}\right)+c_{1}\left(L_{2}\right)$ implies $F_{H}=\mathbb{G}_{\mathrm{a}}$.

Example 3.6. Topological $K$-theory $E^{*}=K^{*}$ on compact spaces is the group completion of isomorphism classes of complex vector bundles. Write $\mathcal{L}_{m}=\left.\mathcal{L}\right|_{\mathbb{C P}^{m}}$ for the tautological complex line bundle over $\mathbb{C P}^{m}, \mathbb{C}$ for the trivial bundle, and $\left[\mathcal{L}_{m}\right], 1 \in K^{0}\left(\mathbb{C P}^{m}\right)$ for their classes in $K$-theory. The classes $\left[\mathcal{L}_{m}\right]-1 \in \widetilde{K}^{0}\left(\mathbb{C P}^{m}\right)$ are compatible under restriction and define a complex orientation $\xi_{K} \in \widetilde{K}^{2}\left(\mathbb{C P}^{\infty}\right)=$ $\lim \widetilde{K}^{0}\left(\mathbb{C P}^{m}\right)$. Here $F_{K}=\mathbb{G}_{\mathrm{m}}$ is the multiplicative formal group law, as

$$
\mu^{*}([\mathcal{L}]-1)=\left[\mu^{*}(\mathcal{L})\right]-1=\left[\pi_{1}^{*}(\mathcal{L}) \otimes \pi_{2}^{*}(\mathcal{L})\right]-1=\mathbb{G}_{\mathrm{m}}([\mathcal{L}]-1,[\mathcal{L}]-1) .
$$

For a complex vector bundle $V \rightarrow X$ of rank $n$ one has $\pi^{*}(V)=\mathcal{L}_{V} \oplus \mathcal{L}_{V}^{\perp}$ over the projectivization $\pi: \mathbb{P}(V) \rightarrow X$ and $\mathcal{L}_{V}^{\perp}$. The formal power series $\Lambda_{t}([V])=$ $1+[V] t+\left[\Lambda^{2} V\right] t^{2}+\ldots \in K^{0}(X) \llbracket t \rrbracket$ has inverse $\Lambda_{-t}([V])$, so $\Lambda_{t}([V]-[W])=$ $\Lambda_{t}([V]) \Lambda_{-t}([W])$. As $\left[\mathcal{L}_{V}^{\perp}\right]=\pi^{*}[V]-[\mathcal{L}]$ has rank $n-1$, the $n$-th coefficient of $\Lambda_{t}\left(\left[\mathcal{L}_{V}^{\perp}\right]\right)=\Lambda_{t}\left(\left[\pi^{*}(V)\right]\right) \Lambda_{-t}(\mathcal{L})$ is $0=\left[\Lambda^{n}\left(\mathcal{L}_{V}^{\perp}\right)\right]=\sum_{p=0}^{n}(-1)^{n-p}\left[\Lambda^{p}(V)\right] \cdot[\mathcal{L}]^{n-p}$. Putting $[\mathcal{L}]=w+1$ and comparing to (3.4), $c_{i}^{K}(V)=\sum_{p=0}^{i}(-1)^{i+p}\left(\begin{array}{l}n-p \\ n-i\end{array}\right)\left[\Lambda^{p}(V)\right]$.

Example 3.7. As in Quillen [18, complex cobordism $\Omega_{\mathrm{U}}^{n}(X)$ for $X$ a smooth manifold is the set of smooth maps $f: Z \rightarrow X$ of codimension $\operatorname{dim} X-\operatorname{dim} Z=$ $n$ with a complex structure the stable normal bundle, modulo cobordism. The complex orientation $\xi_{\Omega} \in \Omega_{\mathrm{U}}^{2}\left(\mathbb{C P}^{\infty}\right)=\lim \Omega_{\mathrm{U}}^{2}\left(\mathbb{C P}^{m}\right)$ is given by $\mathbb{C P}^{m-1} \hookrightarrow \mathbb{C P}^{m}$, and $\mathbb{C P}^{m-1}$ is the zero set of a section of $\mathcal{L}_{m}^{*}$. So for complex line bundles $c_{1}^{\Omega_{\mathrm{U}}}(L)$ is represented by the zero set $s^{-1}(0)$ of a generic section $s: X \rightarrow L$. The formal group law is the universal law $\mathbb{G}_{\mathrm{u}}$, see Adams [1, Part I, §8]. 
Lemma 3.8. Let $V \rightarrow X$ be a complex vector bundle over a finite $C W$ complex. Then each of the Conner-Floyd Chern classes $c_{i}^{E}(V)$ is nilpotent.

Proof. There is a finite open cover $X=\bigcup_{\lambda=1}^{N} U_{\lambda}$ with $U_{\lambda}$ contractible and $\left.V\right|_{U_{\lambda}}$ trivial. From the long exact sequence of the pair $\left(X, U_{\lambda}\right)$ we see that we may lift $c_{i}^{E}(V)$ along $j_{\lambda}^{*}: E^{2 i}\left(X, U_{\lambda}\right) \rightarrow E^{2 i}(X)$ to a class $x_{\lambda} \in E^{2 i}\left(X, U_{\lambda}\right)$. The diagram

$$
\begin{aligned}
& \prod_{\lambda=1}^{N} E^{2 i}\left(X, U_{\lambda}\right) \stackrel{\cup}{\longrightarrow} E^{2 i N}\left(X, \bigcup_{\lambda=1}^{N} U_{\lambda}\right)=E^{2 i N}(X, X)=\{0\} \\
& \downarrow \prod_{\lambda=1}^{N} j_{\lambda}^{*} \quad j^{*} \\
& \prod_{\lambda=1}^{N} E^{2 i}(X) \stackrel{\cup}{\longrightarrow} E^{2 i N}(X)
\end{aligned}
$$

commutes by naturality of ' $\cup$ ', so $c_{i}^{E}(V)^{N}=\prod_{\lambda=1}^{N} j_{\lambda}^{*}\left(x_{\lambda}\right)=j^{*}\left(\prod_{\lambda=1}^{N} x_{\lambda}\right)=0$.

Notation 3.9. When $X$ is a finite CW complex, it follows that we may substitute $w$ by $c_{1}^{E}(L)$ in the formal group law $F(z, w)$. To define the right hand side of (1.4) also for infinite CW complexes $X$, let $\left\{X_{i} \mid i \in I\right\}$ be the direct system of finite subcomplexes $X_{i} \subset X$ ordered by inclusion. The pro-group E-cohomology is the inverse limit $\hat{E}^{*}(X)=\lim E^{*}\left(X_{i}\right)$. The family of all restrictions $F\left(z, c_{1}^{E}\left(\left.L\right|_{X_{i}}\right)\right)$ determines an element we write $F\left(z, c_{1}^{E}(L)\right) \in \hat{E}^{*}(X) \llbracket z \rrbracket$. As homology and direct limits commute, see [20, Prop. 7.53], we have $E_{*}(X)=\operatorname{colim} E_{*}\left(X_{i}\right)$ and therefore a well-defined cap product $E_{*}(X) \otimes \hat{E}^{*}(X) \rightarrow E_{*}(X)$. This defines (1.4) in general.

\section{Proof of Theorem 1.1}

Step 1: Vector bundles over finite $\mathbf{C W}$ complexes. For a complex line bundle $L \rightarrow X$ over a finite CW complex $X$ define $C_{z}^{E}(L)=F\left(z, c_{1}^{E}(L)\right)$. For $V \rightarrow X$ a rank $n$ complex vector bundle we proceed by the splitting principle. As in Definition 3.4 over the projectivization $p: \mathbb{P}(V) \rightarrow X$ we can split off a line bundle from $p^{*}(V)$ and $p^{*}: E^{*}(Y) \rightarrow E^{*}(X)$ is injective. Iterating, we find $q: Y \rightarrow X$ and line bundles $L_{1}, \ldots, L_{n} \rightarrow Y$ with $L_{1} \oplus \cdots \oplus L_{n}=q^{*}(V)$ and $q^{*}: E^{*}(Y) \rightarrow E^{*}(X)$ is injective. By (3.5), the class $q^{*}\left(c_{k}^{E}(V)\right)$ is the $k$-th elementary symmetric polynomial in the Chern roots $c_{1}^{E}\left(L_{1}\right), \ldots, c_{1}^{E}\left(L_{1}\right)$. As the expression

$$
F\left(z, c_{1}^{E}\left(L_{1}\right)\right) \cup \cdots \cup F\left(z, c_{1}^{E}\left(L_{n}\right)\right)=q^{*}\left(C_{z}^{E}(V)\right)
$$

is a symmetric polynomial in the Chern roots, the fundamental theorem of symmetric polynomials implies it has a (unique) preimage $C_{z}^{E}(V)$ in $E^{*}(X) \llbracket z \rrbracket$. The map (1.1) is obtained by combining the class $C_{z}^{E}(V)$ with the cap product

$$
\cap: E_{*}(X) \otimes E^{*}(X) \llbracket z \rrbracket \rightarrow E_{*}(X) \llbracket z \rrbracket .
$$

(a) For naturality, let $f: X^{\prime} \rightarrow X$ and use the pullback $Q: Y^{\prime}=X^{\prime} \times_{X} Y \rightarrow X^{\prime}$ with its canonical map $F: Y^{\prime} \rightarrow Y$ to split $V^{\prime}=f^{*}(V)$ as $Q^{*}\left(V^{\prime}\right) \cong F^{*} q^{*}(V) \cong$ $F^{*}\left(L_{1}\right) \oplus \cdots \oplus F^{*}\left(L_{n}\right)$. Naturality of the Conner-Floyd Chern classes implies that the pullback $F^{*} q^{*}\left(C_{z}^{E}(V)\right)=Q^{*} f^{*}\left(C_{z}^{E}(V)\right)$ of (4.1) along $F$ is $Q^{*} C_{z}^{E}\left(V^{\prime}\right)$. Thus,

$$
C_{z}^{E}\left(f^{*}(V)\right)=f^{*}\left(C_{z}^{E}(V)\right) \text {. }
$$

(b) Let $V, W \rightarrow X$ be vector bundles. Pick $q: Y \rightarrow X$ such that both $q^{*}(V)=$ $L_{1} \oplus \cdots \oplus L_{n}$ and $q^{*}(W)=S_{1} \oplus \cdots \oplus S_{m}$ split into line bundles with $q^{*}$ injective. Then $q^{*} C_{z}^{E}(V)$ equals (4.1), $q^{*} C_{z}^{E}(W)=F\left(z, c_{1}^{E}\left(S_{1}\right)\right) \cup \cdots \cup F\left(z, c_{1}^{E}\left(S_{m}\right)\right)$, and

$$
q^{*} C_{z}^{E}(V \oplus W)=F\left(z, c_{1}^{E}\left(L_{1}\right)\right) \cup \cdots \cup F\left(z, c_{1}^{E}\left(S_{m}\right)\right)=q^{*} C_{z}^{E}(V) \cup q^{*} C_{z}^{E}(W) .
$$

Hence

$$
C_{z}^{E}(V \oplus W)=C_{z}^{E}(V) \cup C_{z}^{E}(W) .
$$

This proves that cap product with $C_{z}^{E}(V)$ satisfies Theorem 1.1(a)\&(b). Part (c) holds by construction. For (d), in the case of line bundles the operation 
$(-) \cap F\left(z, c_{1}^{E}(L)\right)=\sum_{i, j \geqslant 0} F_{i j} z^{i}\left[(-) \cap c_{1}^{E}(L)^{j}\right]$ has degree -2 , as $F_{i j} \in R_{2 i+2 j-2}$. It then follows from (4.1) that in general $(-) \cap C_{z}^{E}(V)$ has degree $-2 \operatorname{rk}(V)$.

(e) Let $V \rightarrow X$ be a vector bundle, $L \rightarrow X$ a complex line bundle, and suppose $q^{*}(V)$ splits as above. Then $q^{*}(L \otimes V)=\left(q^{*}(L) \otimes L_{1}\right) \oplus \cdots \oplus\left(q^{*}(L) \otimes L_{n}\right)$ and so

$$
\begin{aligned}
q^{*} C_{z}^{E}(L \otimes V) & =F\left(z, c_{1}^{E}\left(q^{*}(L) \otimes L_{1}\right)\right) \cup \cdots \cup F\left(z, c_{1}^{E}\left(q^{*}(L) \otimes L_{n}\right)\right) \\
& \stackrel{\sqrt{3.6)}}{=} F\left(z, F\left(q^{*} c_{1}^{E}(L), c_{1}^{E}\left(L_{1}\right)\right)\right) \cup \cdots \cup F\left(z, F\left(q^{*} c_{1}^{E}(L), c_{1}^{E}\left(L_{n}\right)\right)\right) \\
& \stackrel{(2.1)}{=} F\left(F\left(z, q^{*} c_{1}^{E}(L)\right), c_{1}^{E}\left(L_{1}\right)\right) \cup \cdots \cup F\left(F\left(z, q^{*} c_{1}^{E}(L)\right), c_{1}^{E}\left(L_{n}\right)\right) \\
& =q^{*} C_{F\left(z, c_{1}^{E}(L)\right)}^{E}(V) .
\end{aligned}
$$

Hence

$$
C_{z}^{E}(L \otimes V)=C_{F\left(z, c_{1}^{E}(L)\right)}^{E}(V) .
$$

Step 2: Extension to K-theory of finite CW complexes. So far, we have constructed a homomorphism $C_{z}^{E}:(\operatorname{Vect}(X), \oplus) \rightarrow\left(E^{*}(X) \llbracket z \rrbracket, \cup\right)$ on the monoid of complex vector bundles $V \rightarrow X$ up to isomorphism over a finite CW complex. We claim that every class $C_{z}^{E}(V)$ is invertible in the larger ring $E^{*}(X) \llbracket z \rrbracket\left[z^{-1}\right]$. Indeed, there exists a vector bundle $W \rightarrow X$ with $V \oplus W \cong \underline{\mathbb{C}}^{N}$ trivial and therefore $C_{z}^{E}(V) \cup C_{z}^{E}(W)=C_{z}^{E}\left(\underline{\mathbb{C}}^{N}\right)=F\left(z, c_{1}^{E}(\underline{\mathbb{C}})\right)^{N}=z^{N}$. As $X$ is a finite CW complex, its topological $K$-theory is the group completion of $(\operatorname{Vect}(X), \oplus)$ whose universal property allows us to uniquely extend the homomorphism to $C_{z}^{E}: K^{0}(X) \rightarrow$ $\left(E^{*}(X) \llbracket z \rrbracket\left[z^{-1}\right], \cup\right)$. It is easy to check that properties (a)-(d) continue to hold.

Notation 4.1. As $X$ is a finite CW complex, we may write $\theta=[V]-\left[\mathbb{C}^{\ell}\right]$. Expand $C_{z}^{E}(V)=\sum_{n \geqslant 0}^{\infty} C_{n}(V) z^{n}$. Then

$$
C_{z}^{E}(\theta)=\sum_{n \geqslant 0} C_{n}(V) z^{n-\ell}
$$

In Notation 2.1 we have defined $i_{z, w}\left(F(z, w)^{-\ell} \sum_{n \geqslant-\ell}^{\infty} C_{n}(V) F(z, w)^{n}\right)$ as a holomorphic series in $w$ which we can substitute by the nilpotent $c_{1}^{E}(L)$, see Lemma 3.8 , This defines $i_{z, c_{1}^{E}(L)} C_{F\left(z, c_{1}^{E}(L)\right)}^{E}(\theta) \in E^{*}(X) \llbracket z \rrbracket\left[z^{-1}\right]$ for finite $X$. When $X$ is infinite, the classes for the restrictions of $\theta$ to all finite subcomplexes $X_{i} \subset X$ define $i_{z, c_{1}^{E}(L)} C_{F\left(z, c_{1}^{E}(L)\right)}^{E}(\theta) \in \hat{E}(X)((z))$ in pro-group E-cohomology, see Notation 3.9 .

We prove (e). As just seen, $C_{z}^{E}(L)=F\left(z, c_{1}^{E}(L)\right)$ is invertible in $E^{*}(X) \llbracket z \rrbracket\left[z^{-1}\right]$. Therefore $i_{z, w} F\left(z, c_{1}^{E}(L)\right)^{n}=F\left(z, c_{1}^{E}(L)\right)^{n}$ for all $n \in \mathbb{Z}$. Using Notation 4.1 ,

$$
\begin{aligned}
C_{z}^{E}(L \otimes \theta) & \stackrel{\text { (4.3) }}{=} C_{z}(L \otimes V) C_{z}(L)^{-\ell} \\
& \stackrel{\stackrel{4.4}{=}}{=} C_{F\left(z, c_{1}^{E}(L)\right)}(V) F\left(z, c_{1}^{E}(L)\right)^{-\ell} \\
& =\sum_{n \geqslant 0} C_{n}(V) F\left(z, c_{1}^{E}(L)\right)^{n-\ell}=i_{z, c_{1}^{E}(L)} C_{F\left(z, c_{1}^{E}(L)\right)}^{E}(\theta) .
\end{aligned}
$$

Step 3: Infinite complexes. Let $\left\{X_{i} \mid i \in I\right\}$ be the direct system of finite subcomplexes of a CW complex $X$ ordered by inclusion. Write $\iota(i): X_{i} \subset X$ and $\iota(i, j): X_{i} \subset X_{j}$ for the inclusions. For $\theta \in K^{0}(X)$, Step 2 yields maps

$$
E_{*}\left(X_{i}\right) \stackrel{\cap C_{z}\left(\iota(i)^{*} \theta\right)}{\longrightarrow} E_{*}\left(X_{i}\right) \llbracket z \rrbracket\left[z^{-1}\right] \stackrel{\iota(i)_{*}}{\longrightarrow} E_{*}(X) \llbracket z \rrbracket\left[z^{-1}\right] .
$$

By naturality, $\iota(i, j)_{*}(a) \cap C_{z}^{E}\left(\iota(j)^{*} \theta\right)=\iota(i, j)_{*}\left(a \cap C_{z}^{E}\left(\iota(i)^{*} \theta\right)\right)$ so the maps (4.6) determine a homomorphism $E_{*}(X) \cong \operatorname{colim} E_{*}\left(X_{i}\right) \rightarrow E_{*}(X) \llbracket z \rrbracket\left[z^{-1}\right]$ on the colimit, using that homology and direct limits commute, see [20, Prop. 7.53]. Equivalently, the restrictions $C_{z}^{E}\left(\left.\theta\right|_{X_{i}}\right)$ define a class $C_{z}^{E}(\theta) \in \hat{E}^{*}(X)((z))$ in pro-group 
$E$-cohomology. Using the cap product $E_{*}(X) \otimes \hat{E}^{*}(X)((z)) \rightarrow E_{*}(X)((z))$ we can define $(-) \cap C_{z}^{E}(\theta): E_{*}(X) \rightarrow E_{*}(X)((z))$ which, a priori, has a larger codomain.

Finally, properties (a)-(e) pass to the limit.

Step 5: General topological spaces. By the CW approximation theorem, there is a CW complex $X^{\prime}$ with a weak homotopy equivalence $f: X^{\prime} \rightarrow X$. Then

$$
a \cap C_{z}(\theta)=f_{*}\left(f_{*}^{-1}(a) \cap C_{z}\left(f^{*} \theta\right)\right)
$$

is well-defined, since this equation holds for a homotopy equivalence $f: X^{\prime} \rightarrow X^{\prime}$ by (1.2). With this definition, the properties (a)-(e) carry over to $X$.

\section{Proof of Theorem 1.2}

We verify Definition 2.7(a)-(c) for the graded module $V_{*}=\bigoplus E_{*-\operatorname{rk} \theta_{\alpha, \alpha}}\left(X_{\alpha}\right)$, vacuum vector $\Omega=e_{*}(1), F$-shift operator (3.3), and state-to-field correspondence (1.13). Here, $e: \mathrm{pt} \rightarrow X_{0}$ is the H-space unit and $1 \in E_{0}(\mathrm{pt})=R^{0}$.

Writing $|a|_{V}=|a|+\operatorname{rk} \theta_{\alpha, \alpha}$ for the shifted degree, we have

$$
|Y(a, z) b|_{V}=|Y(a, z, b)|+\operatorname{rk} \theta_{\alpha+\beta, \alpha+\beta}=\left(|a|-\operatorname{rk} \theta_{\alpha, \alpha}\right)\left(|b|-\operatorname{rk} \theta_{\beta, \beta}\right)=|a|_{V} \cdot|b|_{V},
$$

so that $Y$ preserves the grading of $V_{*}$.

(a) Let $a \in E_{*}\left(X_{\alpha}\right), b \in E_{*}\left(X_{\beta}\right)$. As $e$ is a fixed point, $\Psi_{*}\left(t_{k} \otimes \Omega\right)=0$ for $k>0$ and $\Psi_{*}\left(t_{0} \otimes \Omega\right)=\Omega$. Hence $\mathcal{D}(z) \Omega=\Omega$. Let $\varphi=\left(e, \operatorname{id}_{X_{\beta}}\right): X_{\beta} \rightarrow X_{\Omega} \times X_{\beta}$. Then

$$
\begin{aligned}
(\Omega \bigotimes b) \cap C_{z}^{E}\left(\theta_{\Omega, \beta}\right) & =\varphi_{*}(b) \cap C_{z}^{E}\left(\theta_{\Omega, \beta}\right) \\
& =\varphi_{*}\left(b \cap \varphi^{*} C_{z}^{E}\left(\theta_{\Omega, \beta}\right)\right) \stackrel{(1.10)}{=} \varphi_{*}(b \cap 1)=\Omega \bigotimes b,
\end{aligned}
$$

and so $Y(\Omega, z) b=\left(\Phi_{\Omega, \beta}\right)_{*}(\mathcal{D}(z) \Omega \otimes b)=b$, proving (2.7). Similarly,

$$
Y(a, z) \Omega=\left(\Phi_{\alpha, \Omega}\right)_{*}\left(\mathcal{D}(z) \otimes \operatorname{id}_{X_{\Omega}}\right)(a \otimes \Omega)=\mathcal{D}(z)(a)
$$

is holomorphic with $\mathcal{D}(0)(a)=a$ for $z=0$, proving (2.6).

(b) We have already shown $\mathcal{D}(z) \Omega=\Omega$. To prove (2.8), we first need a lemma.

Lemma 5.1. For the universal complex line bundle $\mathcal{L} \rightarrow \mathbb{C P}^{\infty}$ and $n \in \mathbb{Z}$

$$
\sum_{k \geqslant 0} t_{k} \cap i_{z, c_{1}^{E}(\mathcal{L})} F\left(z, c_{1}^{E}(\mathcal{L})\right)^{n} w^{k}=\sum_{\ell \geqslant 0} t_{\ell} i_{z, w} F(z, w)^{n} w^{\ell} .
$$

Moreover, for all $a \in E_{*}\left(X_{\alpha}\right), b \in E_{*}\left(X_{\beta}\right)$ we have

$$
\begin{aligned}
& \left(\mathcal{D}_{\alpha}(w) a \otimes b\right) \cap C_{z}^{E}\left(\theta_{\alpha, \beta}\right)=\left(\mathcal{D}_{\alpha}(w) \times \operatorname{id}_{X_{\beta}}\right)\left[(a \otimes b) \cap i_{z, w} C_{F(z, w)}^{E}\left(\theta_{\alpha, \beta}\right)\right], \\
& \left(a \otimes \mathcal{D}_{\beta}(w) b\right) \cap C_{z}^{E}\left(\theta_{\alpha, \beta}\right)=\left(\operatorname{id}_{X_{\alpha}} \times \mathcal{D}_{\beta}(w)\right)\left[(a \otimes b) \cap i_{z, w} C_{F(z, \iota(w))}^{E}\left(\theta_{\alpha, \beta}\right)\right] .
\end{aligned}
$$

Proof. Introduce the expansion $i_{z, w} F(z, w)^{n}=\sum_{i \in \mathbb{Z}, j \geqslant 0} F_{i j}^{n} z^{i} w^{j}$. Then

$$
t_{k} \cap i_{z, c_{1}^{E}(\mathcal{L})} F\left(z, c_{1}^{E}(\mathcal{L})\right)^{n}=t_{k} \cap \sum_{\substack{i \in \mathbb{Z} \\ j \geqslant 0}} F_{i j}^{n} z^{i} c_{1}^{E}(\mathcal{L})^{j}=\sum_{\substack{i \in \mathbb{Z} \\ j \geqslant 0}} F_{i j}^{n} z^{i} t_{k-j}
$$

where $t_{k}=0$ for $k<0$. Summing (5.4) over all $k$, the summands with $k<j$ vanish, so we may restrict the sum to $k \geqslant j$ and reindexing by $\ell=k-j$ gives (5.1):

$$
\sum_{\substack{i \in \mathbb{Z} \\ j \geqslant 0}} \sum_{\ell \geqslant 0} F_{i j}^{n} z^{i} w^{j} t_{\ell} w^{\ell}=\sum_{\ell \geqslant 0} t_{\ell} i_{z, w} F(z, w)^{n} w^{\ell}
$$


For (5.2) we compute

$$
\begin{aligned}
& \left(\mathcal{D}_{\alpha}(w) a \otimes b\right) \cap C_{z}^{E}\left(\theta_{\alpha, \beta}\right) \stackrel{(3.3)}{=} \sum_{k \geqslant 0}\left(\Psi_{\alpha} \times \operatorname{id}_{X_{\beta}}\right)_{*}\left(t_{k} \otimes a \otimes b\right) \cap C_{z}^{E}\left(\theta_{\alpha, \beta}\right) w^{k} \\
& =\left(\Psi_{\alpha} \times \operatorname{id}_{X_{\beta}}\right)_{*} \sum_{k \geqslant 0}\left(t_{k} \otimes a \otimes b\right) \cap\left(\Psi_{\alpha} \times \operatorname{id}_{X_{\beta}}\right)^{*} C_{z}^{E}\left(\theta_{\alpha, \beta}\right) w^{k} \\
& \stackrel{(1.8)}{=}\left(\Psi_{\alpha} \times \operatorname{id}_{X_{\beta}}\right)_{*} \sum_{k \geqslant 0}\left(t_{k} \otimes a \otimes b\right) \cap C_{z}^{E}\left(\mathcal{L} \otimes \theta_{\alpha, \beta}\right) w^{k} \\
& \stackrel{(1.5)}{=}\left(\Psi_{\alpha} \times \operatorname{id}_{X_{\beta}}\right)_{*} \sum_{k \geqslant 0}\left(t_{k} \otimes a \otimes b\right) \cap i_{z, c_{1}^{E}(\mathcal{L})} C_{F\left(z, c_{1}^{E}(\mathcal{L})\right)}^{E}\left(\theta_{\alpha, \beta}\right) w^{k} \\
& \stackrel{(5.1)}{=}\left(\Psi_{\alpha} \times \operatorname{id}_{X_{\beta}}\right)_{*} \sum_{\ell \geqslant 0}\left(t_{\ell} \otimes a \otimes b\right) \cap i_{z, w} C_{F(z, w)}^{E}\left(\theta_{\alpha, \beta}\right) w^{\ell} \\
& =\left(\mathcal{D}_{\alpha}(w) \times \operatorname{id}_{X_{\beta}}\right)\left[(a \otimes b) \cap i_{z, w} C_{F(z, w)}^{E}\left(\theta_{\alpha, \beta}\right)\right] .
\end{aligned}
$$

For (5.3) we similarly use (1.9) which replaces $c_{1}^{E}(\mathcal{L})$ by its formal inverse $\iota\left(c_{1}^{E}(\mathcal{L})\right)$ above, so the same argument with $F(z, \iota(w))$ in place of $F(z, w)$ gives (5.3).

It is now easy to verify (2.8): Let $a \in E_{*}\left(X_{\alpha}\right), b \in E_{*}\left(X_{\beta}\right)$. Then

$$
\begin{aligned}
& Y\left(\mathcal{D}_{\alpha}(w) a, z\right) b \stackrel{(1.13)}{=}\left(\Phi_{\alpha, \beta}\right)_{*}\left(\mathcal{D}_{\alpha}(z) \otimes \operatorname{id}_{\beta}\right)\left[\left(\mathcal{D}_{\alpha}(w) a \otimes b\right) \cap C_{z}^{E}\left(\theta_{\alpha, \beta}\right)\right] \\
& \stackrel{(5.2)}{=}\left(\Phi_{\alpha, \beta}\right)_{*}\left(\mathcal{D}_{\alpha}(z) \mathcal{D}_{\alpha}(w) \otimes \operatorname{id}_{\beta}\right)\left[(a \otimes b) \cap i_{z, w} C_{F(z, w)}^{E}\left(\theta_{\alpha, \beta}\right)\right] \\
& \stackrel{\text { (2.4) }}{=} i_{z, w} Y(a, F(z, w)) b .
\end{aligned}
$$

(c) Firstly, $\Phi \circ(\Psi \times \Psi) \circ \delta \simeq \Psi \circ\left(\Phi \times \operatorname{id}_{B \mathrm{U}(1)}\right)$ and $\Delta_{*}\left(t_{k}\right)=\sum_{i+j=k} t_{i} \otimes t_{j}$ imply

$$
\mathcal{D}_{\alpha+\beta}(z)\left(\Phi_{\alpha, \beta}\right)_{*}=\left(\Phi_{\alpha, \beta}\right)_{*}\left(\mathcal{D}_{\alpha}(z) \otimes \mathcal{D}_{\beta}(z)\right) \text {. }
$$

Let $a \in E_{*}\left(X_{\alpha}\right), b \in E_{*}\left(X_{\beta}\right), c \in E_{*}\left(X_{\gamma}\right)$. On the one hand

$$
\begin{aligned}
& Y(Y(a, z) b, w) c=\left(\Phi_{\alpha+\beta, \gamma}\right)_{*}\left(\mathcal{D}_{\alpha+\beta}(w) \otimes \operatorname{id}_{\gamma}\right) \\
& {\left[\left(\Phi_{\alpha, \beta}\right)_{*}\left(\mathcal{D}_{\alpha}(z) \otimes \operatorname{id}_{\beta}\right)\left[(a \bigotimes b) \cap C_{z}^{E}\left(\theta_{\alpha, \beta}\right)\right] \otimes c \cap C_{w}^{E}\left(\theta_{\alpha+\beta, \gamma}\right)\right]} \\
& \stackrel{\text { (5.5) }}{=}\left(\Phi_{\alpha+\beta, \gamma}\right)_{*}\left(\Phi_{\alpha, \beta}\right)_{*}\left(\mathcal{D}_{\alpha}(w) \otimes \mathcal{D}_{\beta}(w) \otimes \operatorname{id}_{\gamma}\right) \\
& \begin{array}{c}
{\left[\left(\mathcal{D}_{\alpha}(z) \otimes \operatorname{id}_{\beta} \otimes \operatorname{id}_{\gamma}\right)\left((a \otimes b \otimes c) \cap C_{z}^{E}\left(\theta_{\alpha, \beta}\right) \cap\left(\Phi_{\alpha, \beta} \times \operatorname{id}_{\gamma}\right)^{*} C_{w}^{E}\left(\theta_{\alpha+\beta, \gamma}\right)\right)\right]} \\
\stackrel{[2.4}{=,(5.2)}\left(\Phi_{\alpha+\beta, \gamma}\right)_{*}\left(\Phi_{\alpha, \beta}\right)_{*}\left(\mathcal{D}_{\alpha}(w) \mathcal{D}_{\alpha}(z) \otimes \mathcal{D}_{\beta}(w) \otimes \operatorname{id}_{\gamma}\right) \\
{\left[(a \otimes b \otimes c) \cap C_{z}^{E}\left(\theta_{\alpha, \beta}\right) \cap i_{w, z} C_{F(w, z)}^{E}\left(\theta_{\alpha, \gamma}\right) \cap C_{w}^{E}\left(\theta_{\beta, \gamma}\right)\right],}
\end{array}
\end{aligned}
$$

and on the other hand

$$
\begin{aligned}
& i_{z, w} Y(a, F(z, w)) Y(b, w) c=i_{z, w}\left(\Phi_{\alpha, \beta+\gamma}\right)_{*}\left(\mathcal{D}_{\alpha}(F(z, w)) \otimes \operatorname{id}_{\beta+\gamma}\right) \\
& {\left[\left(a \otimes\left(\Phi_{\beta, \gamma}\right)_{*}\left(\mathcal{D}_{\beta}(w) \otimes \mathrm{id}_{\gamma}\right)\left[(b \otimes c) \cap C_{w}^{E}\left(\theta_{\beta, \gamma}\right)\right]\right) \cap C_{F(z, w)}^{E}\left(\theta_{\alpha, \beta+\gamma}\right)\right]} \\
& \text { (5.5), 1.7 } i_{z, w}\left(\Phi_{\alpha, \beta+\gamma}\right)_{*}\left(\operatorname{id}_{\alpha} \otimes \Phi_{\beta, \gamma}\right)_{*}\left(\mathcal{D}_{\alpha}(F(z, w)) \otimes \operatorname{id}_{\beta} \otimes \mathrm{id}_{\gamma}\right) \\
& {\left[\left(\operatorname{id}_{\alpha} \otimes \mathcal{D}_{\beta}(w) \otimes \operatorname{id}_{\gamma}\right)\left[(a \otimes b \otimes c) \cap C_{w}^{E}\left(\theta_{\beta, \gamma}\right)\right] \cap C_{F(z, w)}^{E}\left(\theta_{\alpha, \beta}\right) \cap C_{F(z, w)}^{E}\left(\theta_{\alpha, \gamma}\right)\right]} \\
& \stackrel{2.4,}{=}\left(\Phi_{\alpha, \beta+\gamma}\right)_{*}\left(\operatorname{id}_{\alpha} \otimes \Phi_{\beta, \gamma}\right)_{*}\left(\mathcal{D}_{\alpha}(w) \mathcal{D}_{\alpha}(z) \otimes \mathcal{D}_{\beta}(w) \otimes \operatorname{id}_{\gamma}\right) \\
& {\left[(a \otimes b \otimes c) \cap C_{w}^{E}\left(\theta_{\beta, \gamma}\right) \cap C_{z}^{E}\left(\theta_{\alpha, \beta}\right) \cap i_{z, w} C_{F(z, w)}^{E}\left(\theta_{\alpha, \gamma}\right)\right] .}
\end{aligned}
$$

As $Y(Y(a, z) b, w) c$ and $Y(a, F(z, w)) Y(b, w) c$ are both expansions in negative powers of $F(z, w)$ of the same series in different variables, there exist some $N \gg 0$ with $F(z, w)^{N} Y(Y(a, z) b, w) c=F(z, w)^{N} Y(a, F(z, w)) Y(b, w) c$, see (2.3).

The same calculations show that (1.15) is a nonlocal vertex $F$-algebra and that the state-to-field correspondence $\bar{Y}(a, z) b$ preserves the degree shifted by $2 \chi(\alpha, \alpha)$. 
It remains to prove $(-1)^{a b} \bar{Y}(a, z) b=\mathcal{D}_{\alpha+\beta}(z) \bar{Y}(b, \iota(z) a)$. Notice $\sigma^{*}\left(\bar{C}_{z}^{E}\left(\theta_{\alpha, \beta}\right)\right)=$ $\bar{C}_{\iota(z)}^{E}\left(\theta_{\beta, \alpha}\right)$ for the swap $\sigma: X_{\beta} \times X_{\alpha} \rightarrow X_{\alpha} \times X_{\beta}$. Using $\Phi_{\beta, \alpha} \simeq \Phi_{\alpha, \beta} \circ \sigma$ we find

$$
\begin{aligned}
& \mathcal{D}_{\alpha+\beta}(z) \bar{Y}(b, \iota(z)) a=\mathcal{D}_{\alpha+\beta}(z)\left(\Phi_{\beta, \alpha}\right)_{*}\left(\mathcal{D}_{\beta}(\iota(z)) \otimes \operatorname{id}_{\alpha}\right)\left[(b \otimes a) \cap \bar{C}_{\iota(z)}^{E}\left(\theta_{\beta, \alpha}\right)\right] \\
& =\mathcal{D}_{\alpha+\beta}(z)\left(\Phi_{\alpha, \beta}\right)_{*}\left(\operatorname{id}_{\alpha} \otimes \mathcal{D}_{\beta}(\iota(z))\right) \sigma_{*}\left[(b \otimes a) \cap \sigma^{*} \bar{C}_{z}^{E}\left(\theta_{\alpha, \beta}\right)\right] \\
& \stackrel{\text { 5.5 }}{=}\left(\Phi_{\alpha, \beta}\right)_{*}\left(\mathcal{D}_{\alpha}(z) \otimes \operatorname{id}_{\beta}\right)\left[\sigma_{*}(b \otimes a) \cap \bar{C}_{z}^{E}\left(\theta_{\alpha, \beta}\right)\right] \\
& =\left(\Phi_{\alpha, \beta}\right)_{*}\left(\mathcal{D}_{\alpha}(z) \otimes \operatorname{id}_{\beta}\right)\left[(-1)^{a b}(a \bigotimes b) \cap \bar{C}_{z}^{E}\left(\theta_{\alpha, \beta}\right)\right]=(-1)^{a b} \bar{Y}(a, z) b .
\end{aligned}
$$

Remark 5.2. For the additive formal group law $\mathbb{G}_{\mathrm{a}}$ and ordinary homology, this was shown by Joyce [11, Thm. 3.14]. When $X$ is the derived category of a finite quiver or of certain smooth projective complex varieties, then taking $F(X, Y)=$ $X+Y$ in (1.15) gives a (super) lattice vertex algebra [9, Thm. 5.7] [11, Thm. 5.19].

Remark 5.3. A similar construction applies to $\mathrm{H}$-spaces $X$ with $B \mathrm{O}(1)$-actions, the classifying space for real line bundles, and homology with $\mathbb{Z}_{2}$-coefficients. Since $H^{*}(B \mathrm{O}(1))=\mathbb{Z}_{2} \llbracket \xi \rrbracket$ there is a shift operator $\mathcal{D}(u): H_{*}\left(X ; \mathbb{Z}_{2}\right) \rightarrow H_{*}\left(X ; \mathbb{Z}_{2}\right) \llbracket u \rrbracket$ for $u$ a variable of degree -1 . One can then build, just as in Theorem 1.1, an operator $(-) \cap W_{u}(\theta)$ of degree $-\operatorname{rk} \theta_{\alpha, \beta}$, where $\theta_{\alpha, \beta} \in K O\left(X_{\alpha} \times X_{\beta}\right)$, with normalization $a \cap W_{u}(L)=a \cap\left(u+w_{1}(L)\right)$ for the first Stiefel-Whitney class of a real line bundle $L \rightarrow X$. Then $Y(a, z) b=\left(\Phi_{\alpha, \beta}\right)_{*}\left(\mathcal{D}_{\alpha}(u) \otimes \operatorname{id}_{\beta}\right)\left[(a \otimes b) \cap W_{u}\left(\theta_{\alpha, \beta}\right)\right]$ makes $V=H_{*}\left(X ; \mathbb{Z}_{2}\right)$ into a vertex algebra over $\mathbb{Z}_{2}$.

Acknowledgements. The authors thank Dominic Joyce for many discussions and suggestions. They also thank Mikhail Kapranov, Kobi Kremnitzer, Sven Meinhardt, and Konrad Voelkel for helpful conversations.

\section{REFERENCES}

[1] J.F. Adams, Stable homotopy and generalised homology, Chicago Lectures in Math., University of Chicago Press, Chicago, Ill.-London, 1974.

[2] I.I. Anguelova, Super-bicharacter construction of $H_{D}$-quantum vertex algebras, Rep. Math. Phys. 61 (2008), 253-263.

[3] R.E. Borcherds, Quantum vertex algebras, Adv. Stud. Pure Math. 31 (2001), 51-74.

[4] P.E. Conner and E.E. Floyd, The relation of cobordism to K-theories, Lecture Notes in Math. 28, Springer-Verlag, Berlin-New York, 1966.

[5] E. Frenkel and D. Ben-Zvi, Vertex algebras and algebraic curves, Math. Surveys and Monographs 88, A.M.S., Providence, RI, 2004.

[6] I. Frenkel, J. Lepowsky, and A. Meurman, Vertex operator algebras and the Monster, Pure and Applied Mathematics 134, Academic Press, Inc., Boston, MA, 1988.

[7] L. Göttsche, H. Nakajima, K. Yoshioka, K-theoretic Donaldson invariants via instanton counting, Pure Appl. Math. Q. 5, special issue in honor of F. Hirzebruch (2009), 1029-1111.

[8] L. Göttsche, M. Kool, R.A. Williams, Verlinde formulae on complex surfaces I: K-theoretic invariants, arViv:1903.03869 2019.

[9] J. Gross, The homology of moduli stacks of complexes, arXiv:1907.03269 2019.

[10] J. Gross, D. Joyce, and Y. Tanaka, Universal structures in $\mathbb{C}$-linear enumerative invariant theories. I, arXiv:2005.05637 2020.

[11] D. Joyce, Ringel-Hall style vertex algebra and Lie algebra structures on the homology of moduli spaces, http://people.maths.ox.ac.uk/ joyce/hall.pdf.

[12] D. Joyce, Y. Tanaka and M. Upmeier, On orientations for gauge-theoretic moduli spaces, Adv. Math. 362 (2020). arXiv:1811.01096

[13] D. Joyce and M. Upmeier, Canonical orientations for moduli spaces of $G_{2}$-instantons with gauge group $\mathrm{SU}(m)$ or $\mathrm{U}(m)$, arXiv:1811.02405, 2018.

[14] D. Joyce and M. Upmeier, On spin structures and orientations for gauge-theoretic moduli spaces, arXiv:1908.03524 2019.

[15] V.G. Kac, Vertex algebras for beginners, Univ. Lect. Series 10, A.M.S., Providence, RI, 1998.

[16] Y.-H. Kiem and M. Savvas, K-Theoretic Generalized Donaldson-Thomas Invariants, IMRN (2020). doi.org/10.1093/imrn/rnaa097. 
[17] H.-S. Li, Vertex F-algebras and their $\phi$-coordinated modules, J. Pure Appl. Algebra 215 (2011), 1645-1662.

[18] D. Quillen, Elementary proofs of some results of cobordism theory using Steenrod operations, Adv. Math. 7 (1971), 29-56.

[19] Y.B. Rudyak, On Thom spectra, orientability, and cobordism, Springer Monographs in Mathematics, Springer-Verlag, Berlin, 1998.

[20] R.M. Switzer, Algebraic topology - homotopy and homology, Die Grundlehren der math. Wiss. 212, Springer-Verlag, New York-Heidelberg, 1975.

[21] R.P. Thomas, Equivariant K-theory and refined Vafa-Witten invariants, Comm. Math. Phys. 378 (2020), 1451-1500.

[22] B. Toën and M. Vaquié, Moduli of objects in dg-categories, Ann. Sci. Éc. Norm. Sup. 40 (2007), 387-444.

J. Gross, The Mathematical Institute, Radcliffe Observatory Quarter, Woodstock Road, Oxford, OX2 6GG, U.K.

Email: jacob.gross@maths.ox.ac.uk

M. Upmeier, Department of Mathematics, University of Aberdeen, Fraser Noble Building, Elphinstone Rd, Aberdeen, AB24 3UE, U.K.

E-mail: markus.upmeier@abdn.ac.uk. 